\title{
Nanoliposomal irinotecan in combination with leucovorin and 5-fluorouracil in advanced biliary tract cancers
}

\author{
GABRIEL ALLO $^{1}$, AHU DAMLA CAN ${ }^{1}$, ROGER WAHBA ${ }^{2}$, NILS VOGEL ${ }^{1}$, TOBIAS GOESER ${ }^{1}$, \\ FABIAN KÜTTING $^{1}$ and DIRK WALDSCHMIDT ${ }^{1}$
}

\author{
${ }^{1}$ Department for Gastroenterology and Hepatology, Faculty of Medicine; ${ }^{2}$ Department of General, Visceral and \\ Cancer and Transplant Surgery, University Hospital of Cologne, University of Cologne, D-50937 Cologne, Germany
}

Received August 16, 2021; Accepted November 24, 2021

DOI: $10.3892 / \mathrm{mco} .2021 .2485$

\begin{abstract}
Biliary tract cancers (BTC) are rare but aggressive. Due to limited anti-tumor effects of current second- and later-line treatment regimens, novel treatment options are required. Nanoliposomal irinotecan in combination with leucovorin and 5-fluorouracil (FOLFnal-IRI) achieved promising results as a second-line treatment in patients with pancreatic cancer, warranting further investigation in BTC. In the present study, a retrospective analysis of patients receiving FOLFnal-IRI after initial platinum-based chemotherapy for advanced BTC between January 2016 and August 2020 at the University Hospital Cologne (Cologne, Germany) was performed. A total of 11 patients were identified who met the inclusion criteria. A total of 4 patients (36.4\%) were female and the median age was 54 years. The proportion of patients suffering from gallbladder carcinoma, intrahepatic and extrahepatic cholangiocarcinoma was $18.2,63.6$ and 9.1\%, respectively. Furthermore, 7 patients (63.6\%) received FOLFnal-IRI as their second-, $3(27.3 \%)$ as third- and one $(9.1 \%)$ as their fourth-line therapy. The disease control rate was $54.5 \%$ and 3 grade III toxicities were recorded. Progression-free survival and overall survival (OS) after initiation of FOLFnal-IRI was 5.1 and 12.4 months, respectively. OS after initial diagnosis was 24.7 months. FOLFnal-IRI demonstrated promising antitumor potential with an acceptable safety profile as a subsequent therapy regimen in advanced biliary tract malignancies. Further randomized controlled trials of its value as a treatment option for BTC appear justified.
\end{abstract}

Correspondence to: Dr Gabriel Allo, Department for Gastroenterology and Hepatology, Faculty of Medicine, University Hospital of Cologne, University of Cologne, Kerpenerstr. 62, D-50937 Cologne, Germany

E-mail: gabriel.allo@uk-koeln.de

Abbreviations: BTC, biliary tract cancers; CA19-9, carbohydrate antigen 19-9; FOLFnal-IRI, combination of nal-IRI, leucovorin and 5-fluorouracil; nal-IRI, nanoliposomal irinotecan; OS, overall survival; PFS, progression-free survival; RECIST, response evaluation criteria in solid tumors

Key words: biliary tract cancer, liposomal irinotecan, overall survival, progression free survival, second-line chemotherapy

\section{Introduction}

Biliary tract cancers (BTC) are a rare but aggressive tumor entity. They comprise a heterogenous group of individual malignancies arising from the biliary duct or gallbladder. These tumors may be subdivided into intrahepatic, perihilar and distal extrahepatic cholangiocarcinoma, as well as gallbladder carcinoma. They currently account for $<1 \%$ of malignancies. At the same time, their rising incidence and mortality are increasing the requirement for effective treatment options (1-3).

Surgical resection is the only curative treatment modality. The majority of BTCs are diagnosed at an advanced stage, due to unspecific or complete lack of symptoms, leaving a minority suitable for resection $(4,5)$. Even after successful resection relapse rates are high, resulting in 5-year overall survival rates of only $10-35 \%(6,7)$.

Platinum-based systemic chemotherapy is the established first-line treatment for advanced BTC based on the phase-III ABC-02 trial. This study detected an improved overall survival (OS) in patients treated with a combination chemotherapy consisting of gemcitabine and cisplatin vs. gemcitabine alone (OS, 11.7 vs. 8.1 months) (8). However, sustained response remains infrequent and progression rates are high.

Of those patients who progressed under first-line chemotherapy, $25-50 \%$ may be eligible for subsequent lines of treatment (9). The ABC-06 trial demonstrated the beneficial effects of second-line chemotherapy (10). Fluorouracil-based treatment regimens in combination with oxaliplatin or particularly irinotecan are frequently used as second-line chemotherapy (11-13). Outcomes remain poor with a progression-free survival (PFS) and OS between 2.2-3.6 and 6.7-11 months, respectively (13-15).

Nanoliposomal irinotecan (nal-IRI) is a liposomalencapsulated formulation of irinotecan. The tight encapsulation of thousands of active irinotecan molecules within a polyethylene-glycated lipid bilayer vesicle inhibits protein adsorption and subsequent elimination. The liposomal nanoparticles prolong systemic circulation with a slower release of irinotecan molecules. This enhances the anti-tumor activity and prevents high peak plasma levels, resulting in improved tolerability. In addition, the lipophile formulation favors its accumulation in tumor tissue, 
increasing exposure to the tumor cells. After phagocytosis by tumor-associated macrophages, irinotecan is converted to its active up to 1,000-fold more potent metabolite SN-38. It induces double-strand DNA damage during DNA synthesis by inhibiting topoisomerase-I (16-19).

A significant correlation between topoisomerase-I activity and tumor cell sensitivity to $\mathrm{SN}-38$ was previously observed (20). Since topoisomerase-I activity is enhanced in cisplatin-resistant cancer cells, the use of irinotecan appears to be a reasonable choice in subsequent therapy regimens (21). The plasma concentration of unbound platinum is known to decline in a biphasic manner with an initial and secondary half-life of $31.2 \mathrm{~min}$ and $20.1 \mathrm{~h}$, respectively (22).

Nal-IRI combined with leucovorin and 5-fluorouracil (FOLFnal-IRI) achieved notable results in patients with advanced pancreatic cancer as a second-line treatment in the NAPOLI-1 trial (23). These compelling results led to the initiation of clinical studies to assess its performance in firstand second-line treatments of advanced BTC.

At present, evidence for the efficacy of this combination as a second-line treatment for advanced BTCs is scarce. In the present study, the efficacy of a combined chemotherapy of FOLFnal-IRI in advanced BTC previously treated with platinum-based chemotherapy was assessed.

\section{Materials and methods}

Inclusion criteria. Patients were eligible for inclusion if they were 18 years or older with histologically confirmed cholangiocarcinoma or gallbladder cancer and began treatment with nal-IRI in combination with leucovorin and 5-fluorouracil between 01/08/2017 and 01/04/2020 after initial platinum-based chemotherapy at the University Hospital of Cologne (Cologne, Germany). Patients with ampullary tumors were excluded.

Data collection, end-points and follow-up. The data collected were patient demographics, subtype of BTC and stage at diagnosis in accordance with the 'Union Internationale Contre le Cancer' guidelines (24), prior resection, and plasma levels of carbohydrate antigen 19-9 (CA19-9) during treatment. In addition, the type of chemotherapy prior to and after FOLFnal-IRI therapy, as well as the start and end date of FOLFnal-IRI treatment, were documented.

For the assessment of response, computed tomography and magnetic resonance imaging performed during treatment with FOLFnal-IRI were reviewed and response was classified as complete or partial response, or stable or progressive disease according to the radiologist's evaluation.

Toxicity was graded according to the 'Common Terminology Criteria for Adverse Events' (version 5.0) published by the US National Cancer Institute (25). All documented grade III or higher adverse events, as well as alterations of laboratory parameters leading to dose reduction, were analyzed.

Patients were followed up until death or 01/08/2020. OS was defined as the period from the start of FOLFnal-IRI until death from any cause. Data on patients who were alive at the end of the follow-up (01/08/2020) were regarded censored to the date of the last follow-up. PFS was defined as the period from the start of FOLFnal-IRI to the date of a documented disease progression or death.

In accordance with the regional law (paragraph 15, sentence 1, North Rhine Medical Association's Professional Code of Conduct from 14th November 1998, as amended on 16th November 2019, and paragraph 6, sentence 1, Health Data Protection Act of North Rhine Westphalia) (26), approval by a local ethics committee and written informed consent from the participants were not required due to the strictly retrospective design of the study.

Treatment. FOLFnal-IRI was applied according to the NAPOLI-1 trial protocol: Liposomal irinotecan at $70 \mathrm{mg} / \mathrm{m}^{2}$ and folinic acid at $400 \mathrm{mg} / \mathrm{m}^{2}$, followed by 5 -fluorouracil infusion at $2,400 \mathrm{mg} / \mathrm{m}^{2}$ over the course of $46 \mathrm{~h}$ every 2 weeks (23).

Statistical analysis. For descriptive analyses, SPSS version 26 (IBM Corporation) was used. Categorical variables are presented as absolute numbers and relative frequencies. Continuous variables are expressed as the median (range). PFS and OS were estimated from Kaplan-Meier curves.

\section{Results}

Patient characteristics. A total of 14 patients were treated with FOLFnal-IRI during the period of interest. Of these, three patients were excluded from analysis: One patient received only one cycle of FOLFnal-IRI and wished to stop further treatment due to personal reasons. One patient, already hospitalized and moribund due to end-stage BTC and liver failure, received 50\% FOLFnal-IRI off-label as a last resort but died shortly after and one patient was lost to follow-up. Finally, 11 patients were included in the present study. The median age was 54 years (range, 41-69 years). A total of 4 patients were female $(36.4 \%)$. Furthermore, $7(63.6 \%)$ patients were diagnosed with intrahepatic cholangiocarcinoma, 2 (18.2\%) with gallbladder cancer and $1(9.1 \%)$ with extrahepatic cholangiocarcinoma. Surgical resection was performed in $4(36.4 \%)$ patients prior to therapy with FOLFnal-IRI. The initial stage at the start of FOLFnal-IRI administration according to the classification of the 'Union International Contre Le Cancer' was IV in the $11(100 \%)$ patients (all previously resected patients had progressed to stage IV) (Table I).

Treatment. The first-line chemotherapy regimen was platinum-based in all cases. Of the patients, 7 (63.6\%) received FOLFnal-IRI as their second-, $3(27.3 \%)$ as third- and $1(9.1 \%)$ as their fourth-line chemotherapy. Furthermore, one patient received FOLFnal-IRI in combination with trastuzumab. A total of $6(54.6 \%)$ patients received at least one subsequent chemotherapy after FOLFnal-IRI (Table I).

The median duration of treatment with FOLFnal-IRI was 8.7 months (range, 0.9-12 months) (Table II). FOLFnal-IRI was postponed at least once in $5(45.5 \%)$ patients and the dose of applied chemotherapy was reduced at least once in 7 patients $(63.7 \%)$. A total of 3 grade III toxicities were recorded. In two patients, grade III diarrhea led to termination of treatment (data not shown). 
Table I. Patient characteristics $(n=11)$.

\begin{tabular}{lc}
\hline Item & Value \\
\hline Female sex & $4(36.4)$ \\
Age at initial diagnosis, years & $54(41-69)$ \\
Age at start of FOLFnal-IRI, years & $56(44-69)$ \\
Subtype of BTC & \\
Intrahepatic cholangiocarcinoma & $7(63.6)$ \\
Extrahepatic cholangiocarcinoma & $1(9.1)$ \\
Gallbladder carcinoma & $2(18.2)$ \\
Prior resection of primary tumor performed & $4(36.4)$ \\
Stage IV at treatment with & $11(100)$ \\
FOLFnal-IRI & $11(100)$ \\
Initial platinum-based chemotherapy & \\
FOLFnal-IRI as n line of therapy & $7(63.6)$ \\
Second & $3(27.3)$ \\
Third & $1(9.1)$ \\
Fourth & $6(54.6)$ \\
Patients with subsequent lines & \\
of therapy & \\
\hline
\end{tabular}

Values are expressed as n (\%) or median (range). BTC, biliary tract cancer; FOLFnal-IRI, combination of nanoliposomal irinotecan, leucovorin and 5-fluorouracil.

Table II. Outcomes after initiation of FOLFnal-IRI.

\begin{tabular}{lc}
\hline Item & Value \\
\hline Duration of treatment, months & $8.7(0.9-12)$ \\
Best radiological response & \\
Partial response & $0(0)$ \\
Stable disease & $6(54.6)$ \\
Progressive disease & $5(45.5)$ \\
Progression-free survival, months & \\
Total & $5.1(1.1-11.5)$ \\
Second-line & $6.1(1.1-11.5)$ \\
Third-line & $3.9(2.1-10.8)$ \\
Fourth-line & $5.1(-)$ \\
OS after initiation, months & $12.4(3.9-22.2)$ \\
Total & $12.1(5.1-22.2)$ \\
Second-line & $12.4(3.9-14.9)$ \\
Third-line & $16.5(-)$ \\
Fourth-line & $24.7(10.1-65.2)$ \\
OS after initial diagnosis, months & \\
\hline
\end{tabular}

Values are expressed as n (\%) or median (range). FOLFnal-IRI, combination of nanoliposomal irinotecan, leucovorin and 5-fluorouracil; OS, overall survival.

The mean CA19-9 levels at the start and end of FOLFnal-IRI were $309.6 \pm 449.1$ and $708.9 \pm 1891.7 \mathrm{kU} / 1$, respectively. The mean maximum reduction of CA19.9 during treatment was $24.7 \pm 31.7 \%$ (data not shown).

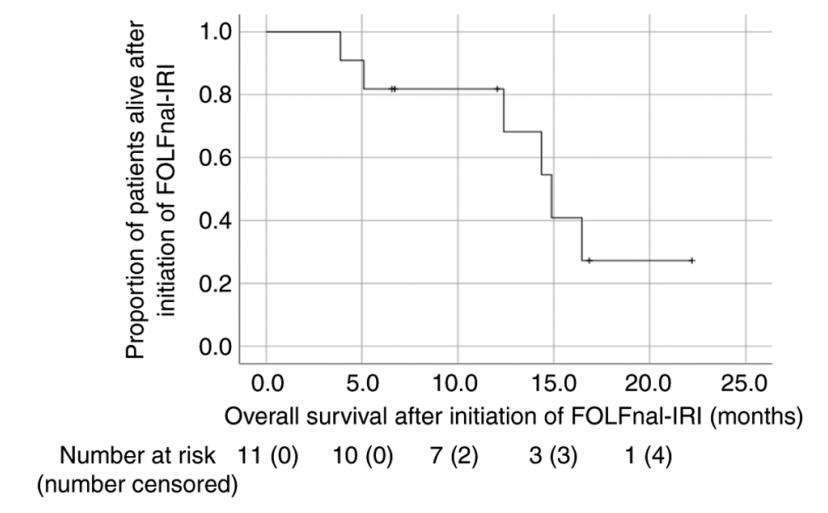

Figure 1. Kaplan-Meier curve of overall survival after initiation of FOLFnal-IRI. FOLFnal-IRI, combination of nanoliposomal irinotecan, leucovorin and 5-fluorouracil.

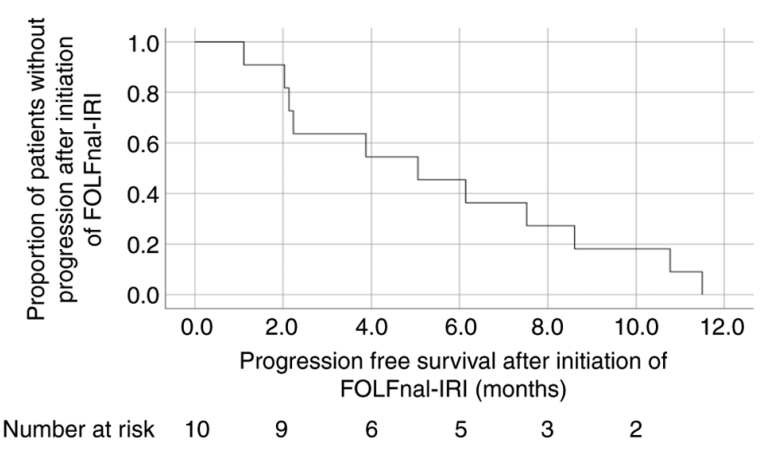

Figure 2. Kaplan-Meier curve of progression-free survival after initiation of FOLFnal-IRI. FOLFnal-IRI, combination of nanoliposomal irinotecan, leucovorin and 5-fluorouracil.

Patient survival. The median PFS and OS from the start of FOLFnal-IRI and OS from initial diagnosis of stage IV BTC were 5.1, 12.4 and 24.7 months, respectively (Figs. 1 and 2; Table II). The median PFS of patients treated with FOLFnal-IRI as second-, third- and fourth-line chemotherapy was $6.1,3.9$ and 5.1 months, respectively. The median OS of patients after the start of FOLFnal-IRI as their second-, thirdand fourth-line chemotherapy was 12.1, 12.4 and 16.5 months, respectively. A total of $5(45.5 \%)$ patients were still alive at the date of censoring. Furthermore, $1(9.1 \%)$ patient died prior to undergoing their subsequent staging examination after the start of treatment. The best documented radiological response was stable disease, which was achieved in 6 patients, resulting in a disease control rate of $54.6 \%$ (Table II).

\section{Discussion}

Patients with advanced BTC only have a small number of established therapeutic options in contrast to those with other tumor entities, e.g. lung cancer, where established options for further lines of treatment positively influence patient survival (27).

In the present study, promising results for FOLFnal-IRI in advanced BTC after failure of initial platinum-based chemotherapy are provided. FOLFnal-IRI had remarkable efficiency with a disease control rate of $54.5 \%$, a median PFS of 5.1 months and OS of 12.4 months. OS after initial diag- 
nosis of stage IV BTC was 24.7 months and toxicity remained modest. At present, only limited data are available on the efficacy of FOLFnal-IRI in advanced biliary tract malignancies.

The analysis of the present study indicated favorable results compared to previous studies evaluating the benefit of second-line chemotherapy in advanced BTC. A large multicenter retrospective study by Brieau et al (13) in 2015 involving patients treated with different second-line regimens recorded a median PFS and OS of 3.2 and 6.7 months, respectively. A meta-analysis of 25 studies provided comparable results for the mean PFS and OS (28). Of note, these studies were not able to demonstrate any difference between regimens with regard to fluorouracil- or gemcitabine-based chemotherapy nor single or combination protocols.

The results of the present study likely reflect the expected benefit of the novel liposomal formula of irinotecan compared to published data from patients with advanced BTC receiving conventional FOLFIRI as a second-line treatment. In these studies, PFS and OS ranged from 2.4 to 3.5 months and from 5.5 to 6.6 months, respectively $(11,29,30)$. Superiority of liposomal irinotecan over standard irinotecan in other malignancies has also been described in previous preclinical and clinical studies $(17,18,31)$.

To the best of our knowledge, only one small retrospective analysis of FOLFnal-IRI in BTC was previously performed (32). This study included 14 patients from Austria and provided exceptional results. The PFS of 10.6 months and OS of 24.1 months after initiation of FOLFnal-IRI as a subsequent line of treatment appear extraordinarily effective when comparing them to the OS of 11.7 months achieved by the combination therapy of gemcitabine and cisplatin as first-line therapy in the ABC-O2-trial (8). The results of the present study relativize the exceptional results from Austria; however, FOLFnal-IRI remains a promising treatment option with regard to limited results of other subsequent treatment regimens in advanced BTC. Due to these nonetheless promising results, further investigation of the value of FOLFnal-IRI as a treatment option in BTC is warranted.

The actual PFS may have been even higher in the present study if the radiologist's evaluation had been based on the RESPONSE EVALUATION CRITERIA IN SOLID TUMORS (RECIST) (33), as it has been in several of the above mentioned studies $(29,30,32)$. Any reported increase in size or quantity was classified as disease progression, whereas progression according to the RECIST criteria is classified as an increase in size of $>20 \%$. Since these criteria are not applied in routine clinical practice at our institution, much higher rates of progressive disease may have been documented in this patient population.

Currently, numerous clinical studies are evaluating FOLFnal-IRI in various disease entities. Of these prospective trials, six investigate its performance in advanced BTC. The phase II NAPOLI-2 trial (no. NCT04005339) evaluates the clinical activity of FOLFnal-IRI in a single-arm setting following gemcitabine and platinum chemotherapy. In 2017, the 'Working Group for Internal Oncology of the German Cancer Society'-associated randomized phase II trial NALIRICC (no. NCT03043547), which compares FOLFnal-IRI to fluorouracil/folinic acid monotherapy in patients who progressed after first-line chemotherapy with gemcitabine-based regi- mens was started. Since 2018, another randomized phase II trial (no. NCT03524508) is comparing FOLFnal-IRI to fluorouracil/folinic acid monotherapy as second-line therapy after failure of initial combination therapy with gemcitabine and cisplatin. In addition, the randomized multicenter phase II trial NIFE (no. NCT03044587) is comparing FOLFnal-IRI with gemcitabine and cisplatin as a first-line treatment in patients with locally advanced or metastatic BTC.

In addition, further improvement of FOLFnal-IRI is under investigation. The NAPOLI-3 trial examines the efficacy of the addition of oxaliplatin to FOLFnal-IRI in pancreatic cancer and potential positive results may translate to further investigation in BTC. Furthermore, novel therapeutic agents are being investigated in combination with FOLFnal-IRI. ACCRU-GI-1603 (no. NCT03337087) is a phase I/II trial testing FOLFnal-IRI in combination with the PARP inhibitor rucaparib in BTC and other gastrointestinal malignancies and simultaneous checkpoint inhibition with nivolumab in combination with FOLFnal-IRI is being investigated in a phase Ib/II trial (no. NCT03785873) as a second-line treatment in advanced BTC. The results of these studies are highly anticipated.

Recently, targeted therapy became an encouraging option for subsequent therapy for BTC with fibroblast growth factor receptor fusion or isocitrate dehydrogenase-1 mutations $(34,35)$. However, merely $10-15 \%$ of BTCs carry such targetable genetic alterations, limiting the impact of these drugs in the overall population of patients with advanced BTC $(35,36)$.

While the results of the present analysis are promising, its limitations should be mentioned. The sample size was relatively small and the study was of a monocentric and retrospective nature. The lack of a control group and proper randomization may have led to survivorship bias, since healthier patients are more likely to receive subsequent lines of chemotherapy after failure of initial treatment regimens. Considering the dismal results of second-line trials from the past in this difficult-to-treat disease, further investigation of the value of FOLFnal-IRI as a treatment option in BTC is worthwhile.

In conclusion, FOLFnal-IRI demonstrated promising antitumor potential with an acceptable safety profile as a subsequent therapy regimen in advanced biliary tract malignancies. Further results of the above-mentioned trials are highly anticipated to determine its potential as a treatment option in this difficult-to-treat entity.

\section{Acknowledgements}

Not applicable.

\section{Funding}

No funding was received.

\section{Availability of data and materials}

The datasets used and/or analyzed during the current study are available from the corresponding author upon reasonable request. 


\section{Authors' contributions}

GA, DW and FK designed the study and drafted the manuscript. GA, ADC and NV acquired data and performed data analysis. FK and DW checked and approved the authenticity of the raw data. GA, DW and FK performed the statistical analysis. TG, RW, FK and DW were involved in the conceptualization, methodology and supervision of the study. All authors read and approved the final manuscript.

\section{Ethics approval and consent to participate}

In accordance with the regional law (paragraph 15, sentence 1, North Rhine Medical Association's professional code of conduct from 14th November 1998 as amended on 16th November 2019, and paragraph 6, sentence 1, Health Data Protection Act of North Rhine-Westphalia) (26), approval by a local ethics committee and written informed consent from the participants were not required due to the strictly retrospective design of the present study.

\section{Patient consent for publication}

Not applicable.

\section{Competing interests}

The authors declare that they have no competing interests.

\section{References}

1. Siegel RL, Miller KD and Jemal A: Cancer statistics, 2019. CA Cancer J Clin 69: 7-34, 2019.

2. von Hahn T, Ciesek S, Wegener G, Plentz RR, Weismüller TJ, Wedemeyer H, Manns MP, Greten TF and Malek NP: Epidemiological trends in incidence and mortality of hepatobiliary cancers in Germany. Scand J Gastroenterol 46: 1092-1098, 2011

3. Saha SK, Zhu AX, Fuchs CS and Brooks GA: Forty-year trends in cholangiocarcinoma incidence in the U.S.: Intrahepatic disease on the rise. Oncologist 21: 594-599, 2016.

4. Matsuo K, Rocha FG, Ito K, D'Angelica MI, Allen PJ, Fong Y, Dematteo RP, Gonen M, Endo I and Jarnagin WR: The blumgart preoperative staging system for hilar cholangiocarcinoma: Analysis of resectability and outcomes in 380 patients. J Am Coll Surg 215: 343-355, 2012.

5. Fong Y, Jarnagin W and Blumgart LH: Gallbladder cancer: Comparison of patients presenting initially for definitive operation with those presenting after prior noncurative intervention. Ann Surg 232: 557-569, 2000.

6. Tamandl D, Herberger B, Gruenberger B, Puhalla H, Klinger M and Gruenberger T: Influence of hepatic resection margin on recurrence and survival in intrahepatic cholangiocarcinoma. Ann Surg Oncol 15: 2787-2794, 2008.

7. Wang Y, Li J, Xia Y, Gong R, Wang K, Yan Z, Wan X, Liu G, Wu D, Shi L, et al: Prognostic nomogram for intrahepatic cholangiocarcinoma after partial hepatectomy. J Clin Oncol 31 1188-1195, 2013.

8. Valle J, Wasan H, Palmer DH, Cunningham D, Anthoney A, Maraveyas A, Madhusudan S, Iveson T, Hughes S, Pereira SP, et al: Cisplatin plus gemcitabine versus gemcitabine for biliary tract cancer. N Engl J Med 362: 1273-1281, 2010.

9. Kim MJ, Oh DY, Lee SH, Kim DW, Im SA, Kim TY, Heo DS and Bang YJ: Gemcitabine-based versusfluoropyrimidine-based chemotherapy with or without platinum in unresectable biliary tract cancer: A retrospective study. BMC Cancer 8: 374, 2008.

10. Lamarca A, Palmer DH, Wasan HS, Ross PH, Ma YT, Arora A, Falk S, Gillmore R, Wadsley J, Patel K, et al: ABC-06 I A randomised phase III, multi-centre, open-label study of active symptom control (ASC) alone or ASC with oxaliplatin/5-FU chemotherapy (ASC+mFOLFOX) for patients (pts) with locally advanced/metastatic biliary tract cancers (ABC) previously-treated with cisplatin/gemcitabine (CisGem) chemotherapy. J Clin Oncol 37: 4003, 2019.
11. Feisthammel J, Schoppmeyer K, Mössner J, Schulze M, Caca K and Wiedmann M: Irinotecan With 5-FU/FA in advanced biliary tract adenocarcinomas. Am J Clin Oncol 30: 319-324, 2007.

12. Guion-Dusserre JF, Lorgis V, Vincent J, Bengrine L and Ghiringhelli F: FOLFIRI plus bevacizumab as a second-line therapy for metastatic intrahepatic cholangiocarcinoma. World J Gastroenterol 21: 2096-2101, 2015.

13. Brieau B, Dahan L, De Rycke Y, Boussaha T, Vasseur P, Tougeron D, Lecomte T, Coriat R, Bachet JB, Claudez P, et al: Second-line chemotherapy for advanced biliary tract cancer after failure of the gemcitabine-platinum combination: A large multicenter study by the association des gastro-entérologues oncologues. Cancer 121: 3290-3297, 2015.

14. Lowery MA, Goff LW, Keenan BP, Jordan E, Wang R, Bocobo AG, Chou JF, O'Reilly EM, Harding JJ, Kemeny N, et al: Second-line chemotherapy in advanced biliary cancers: A retrospective, multicenter analysis of outcomes. Cancer 125 : 4426-4434, 2019.

15. Schweitzer N, Kirstein MM, Kratzel AM, Mederacke YS, Fischer M, Manns MP and Vogel A: Second-line chemotherapy in biliary tract cancer: Outcome and prognostic factors. Liver Int 39: 914-923, 2019.

16. Kalra AV, Kim J, Klinz SG, Paz N, Cain J, Drummond DC, Nielsen UB and Fitzgerald JB: Preclinical activity of nanoliposomal irinotecan is governed by tumor deposition and intratumor prodrug conversion. Cancer Res 74: 7003-7013, 2014.

17. Leonard SC, Lee H, Gaddy DF, Klinz SG, Paz N, Kalra AV, Drummond DC, Chan DC, Bunn PA, Fitzgerald JB and Hendriks BS: Extended topoisomerase 1 inhibition through liposomal irinotecan results in improved efficacy over topotecan and irinotecan in models of small-cell lung cancer. Anticancer Drugs 28: 1086-1096, 2017.

18. Drummond DC, Noble CO, Guo Z, Hong K, Park JW and Kirpotin DB: Development of a highly active nanoliposomal irinotecan using a novel intraliposomal stabilization strategy. Cancer Res 66: 3271-3277, 2006.

19. Kawato Y, Aonuma M, Hirota Y, Kuga H and Sato K: Intracellular roles of SN-38, a metabolite of the camptothecin derivative CPT-11, in the antitumor effect of CPT-11. Cancer Res 51: 4187-4191, 1991 .

20. Kigawa J: New strategy for overcoming resistance to chemotherapy of ovarian cancer. Yonago Acta Med 56: 43-50, 2013.

21. Fukuda M, Nishio K, Kanzawa F, Ogasawara H, Ishida T, Arioka H, Bojanowski K, Oka M and Saijo N: Synergism between cisplatin and topoisomerase I inhibitors, NB-506 and SN-38, in human small cell lung cancer cells. Cancer Res 56: 789-793, 1996.

22. Kitajima K, Fukuoka M, Kobayashi S, Kusunoki Y, Takada M, Negoro S, Matsui K, Sakai N, Ryu S and Takifuji N: Studies on the appropriate administration of cisplatin based on pharmacokinetics and toxicity. Gan To Kagaku Ryoho 14: 2517-2523, 1987 (In Japanese).

23. Wang-Gillam A, Li CP, Bodoky G, Dean A, Shan YS, Jameson G, Macarulla T, Lee KH, Cunningham D, Blanc JF, et al: Nanoliposomal irinotecan with fluorouracil and folinic acid in metastatic pancreatic cancer after previous gemcitabine-based therapy (NAPOLI-1): A global, randomised, open-label, phase 3 trial. Lancet 387: 545-557, 2016.

24. Brierley J,Gospodarowicz MK and Wittekind C: TNMClassification of Malignant Tumours. Wiley Blackwell, Oxford, 2017.

25. US Department of Health and Human Services. National Institutes of Health NCI: Common Terminology Criteria for Adverse Events (CTCAE) Common Terminology Criteria for Adverse Events (CTCAE) v5.0. Accepted November 27, 2017.

26. Ärztekammer Nordrhein: Berufsordnung Für Die Nordrheinischen Ärztinnen und Ärzte. Vom 14. November 1998 in der Fassung vom 16. November 2019 (in Kraft getreten am 4. April 2020), 2019.Available from: https://www.aekno.de/aerzte/ berufsordnung.

27. Nadler E, Espirito JL, Pavilack M, Boyd M, Vergara-Silva A and Fernandes A: Treatment patterns and clinical outcomes among metastatic non-small-cell lung cancer patients treated in the community practice setting. Clin Lung Cancer 19:360-370, 2018.

28. Lamarca A, Hubner RA, David Ryder W and Valle JW: Second-line chemotherapy in advanced biliary cancer: A systematic review. Ann Oncol 25: 2328-2338, 2014.

29. Mizrahi JD, Gunchick V, Mody K, Xiao L, Surapaneni P, Shroff RT and Sahai V: Multi-institutional retrospective analysis of FOLFIRI in patients with advanced biliary tract cancers. World J Gastrointest Oncol 12: 83-91, 2020.

30. Moretto R, Raimondo L, De Stefano A, Cella CA, Matano E, De Placido $S$ and Carlomagno C: FOLFIRI in patients with locally advanced or metastatic pancreatic or biliary tract carcinoma. Anticancer Drugs 24: 980-985, 2013. 
31. Chibaudel B, Maindrault-Gœbel F, Bachet JB, Louvet C, Khalil A, Dupuis O, Hammel P, Garcia ML, Bennamoun M, Brusquant D, et al: PEPCOL: A GERCOR randomized phase II study of nanoliposomal irinotecan PEP02 (MM-398) or irinotecan with leucovorin/5-fluorouracil as second-line therapy in metastatic colorectal cancer. Cancer Med 5: 676-683, 2016.

32. Taghizadeh H, Unseld M, Schmiderer A, Djanani A, Wilthoner K, Buchinger D and Prager GW: First evidence for the antitumor activity of nanoliposomal irinotecan with 5-fluorouracil and folinic acid in metastatic biliary tract cancer. Cancer Chemother Pharmacol 86: 109-115, 2020.

33. Eisenhauer EA, Therasse P, Bogaerts J, Schwartz LH, Sargent D, Ford R, Dancey J, Arbuck S, Gwyther S, Mooney M, et al: New response evaluation criteria in solid tumours: Revised RECIST guideline (version 1.1). Eur J Cancer 45: 228-247, 2009.

34. Abou-Alfa GK, Macarulla T, Javle MM, Kelley RK, Lubner SJ, Adeva J, Cleary JM, Catenacci DV, Borad MJ, Bridgewater J, et al: Ivosidenib in IDH1-mutant, chemotherapy-refractory cholangiocarcinoma (ClarIDHy): A multicentre, randomised, double-blind, placebo-controlled, phase 3 study. Lancet Oncol 21: 796-807, 2020.
35. Abou-Alfa GK, Sahai V, Hollebecque A, Vaccaro G, Melisi D, Al-Rajabi R, Paulson AS, Borad MJ, Gallinson D, Murphy AG, et al: Pemigatinib for previously treated, locally advanced or metastatic cholangiocarcinoma: A multicentre, open-label, phase 2 study. Lancet Oncol 21: 671-684, 2020.

36. Boscoe AN, Rolland C and Kelley RK: Frequency and prognostic significance of isocitrate dehydrogenase 1 mutations in cholangiocarcinoma: A systematic literature review. J Gastrointest Oncol 10: 751-765, 2019.

This work is licensed under a Creative Commons Attribution-NonCommercial-NoDerivatives 4.0 International (CC BY-NC-ND 4.0) License. 